C.W.J. has a financial interest in Global Thermostat Operations, LLC.

\title{
Gasification of radical coke with steam and steam-hydrogen mixtures over manganese-chromium oxides
}

\author{
Maxim P. Bukhovko ${ }^{\mathrm{a}}$, Lu Yang ${ }^{\mathrm{b}}$, Liwei Li ${ }^{\mathrm{c}}$, Andrzej Malek ${ }^{\mathrm{d}}$, Robert J. Davis ${ }^{\mathrm{b}}$, Pradeep K. \\ Agrawal $^{\mathrm{e}^{*}}$, Christopher W. Jones ${ }^{\mathrm{a}^{*}}$ \\ ${ }^{a}$ School of Chemical \& Biomolecular Engineering, Georgia Institute of Technology, Atlanta, GA 30332, USA \\ ${ }^{b}$ Department of Chemical Engineering, University of Virginia, Charlottesville, VA 22904, USA \\ ${ }^{c}$ Hydrocarbons R\&D, The Dow Chemical Company, Freeport, TX 77541, USA \\ ${ }^{d}$ Hydrocarbons R\&D, The Dow Chemical Company, Midland, MI 48674, USA \\ ${ }^{e}$ Department of Chemical Engineering, Michigan Technological University, Houghton, MI 49931, USA \\ *Correspondingauthors:pkagrawa@mtu.edu,christopher.jones@.chbe.gatech.edu
}

\section{Supporting Information}

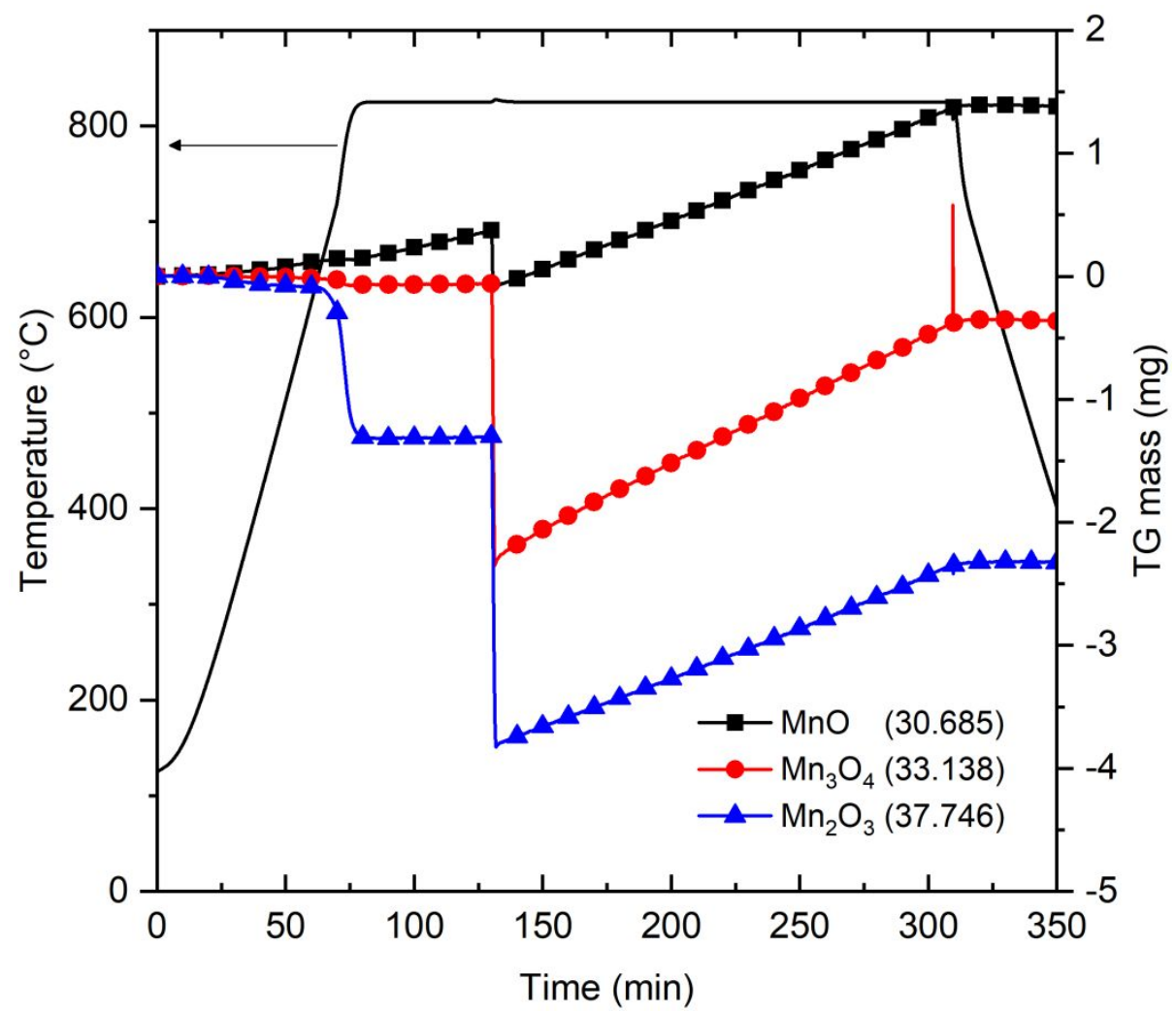

Figure S1. TG profiles of $\mathrm{MnO}_{\mathrm{x}}$ powders during in situ coking procedure. Powders were heated under nitrogen flow and $2 \mathrm{~mol} \%$ ethylene feed was introduced at $t=130 \mathrm{~min}$ for a duration of 3 h. Initial sample masses $(\mathrm{mg})$ are shown in parentheses. 


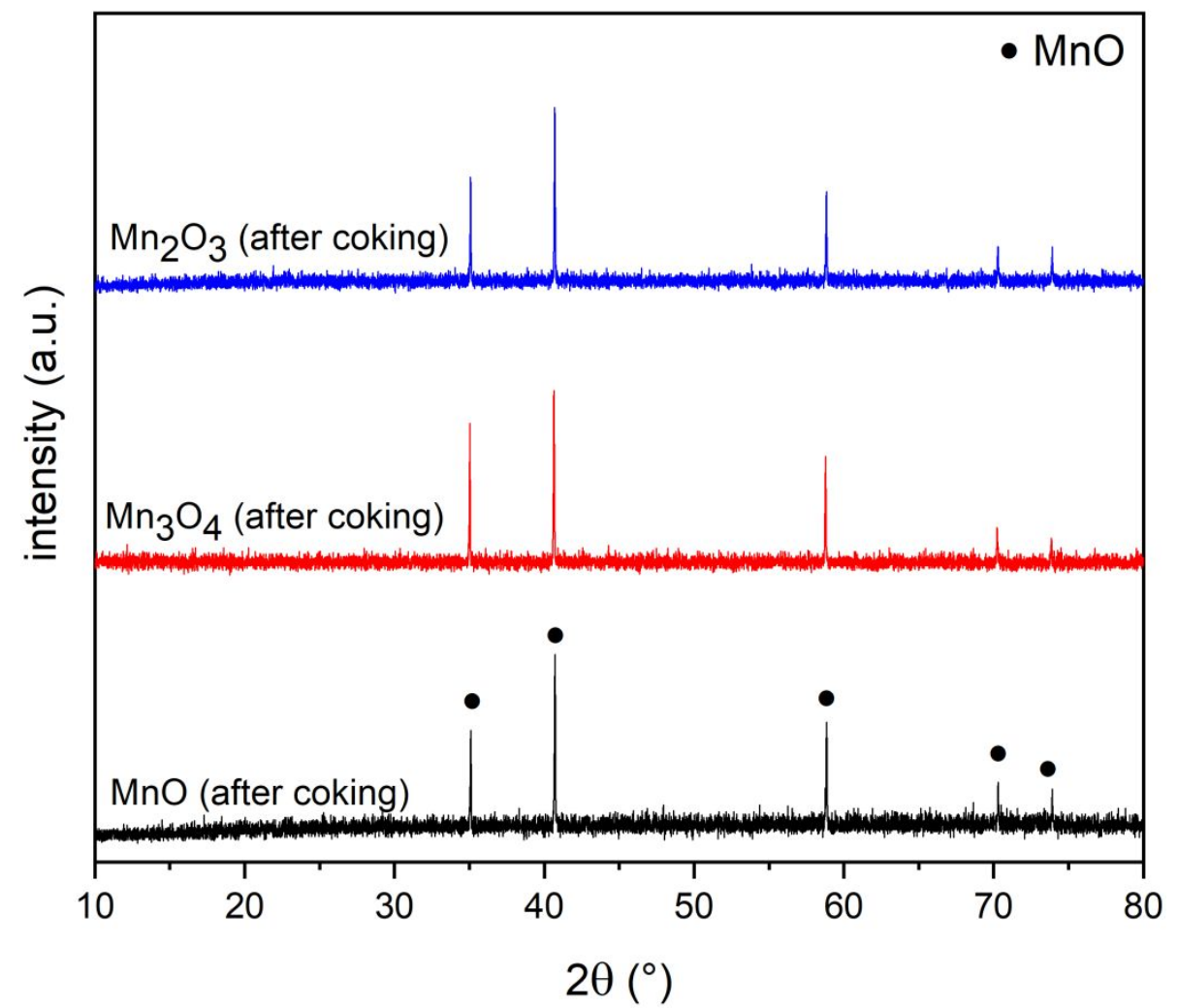

Figure S2. X-ray diffraction patterns of $\mathrm{MnO}_{\mathrm{x}}$ powders after in situ coking with $2 \mathrm{~mol} \%$ ethylene at $825^{\circ} \mathrm{C}$ for $3 \mathrm{~h}$.

Table S1. Bounded parameters for Raman spectra peak deconvolution.

\begin{tabular}{ccccc}
\hline Peak & \multicolumn{2}{c}{ Position $\left(\mathbf{c m}^{-1}\right)$} & \multicolumn{2}{c}{ FWHM $\left(\mathbf{c m}^{-1}\right)$} \\
& lower bound & upper bound & lower bound & upper bound \\
\hline G & 1575 & 1585 & 60 & 85 \\
D1 & 1345 & 1355 & 140 & 200 \\
D2 & 1615 & 1620 & 40 & 65 \\
D3 & 1500 & 1520 & 50 & 300 \\
D4 & 1180 & 1200 & 150 & 300 \\
\hline
\end{tabular}




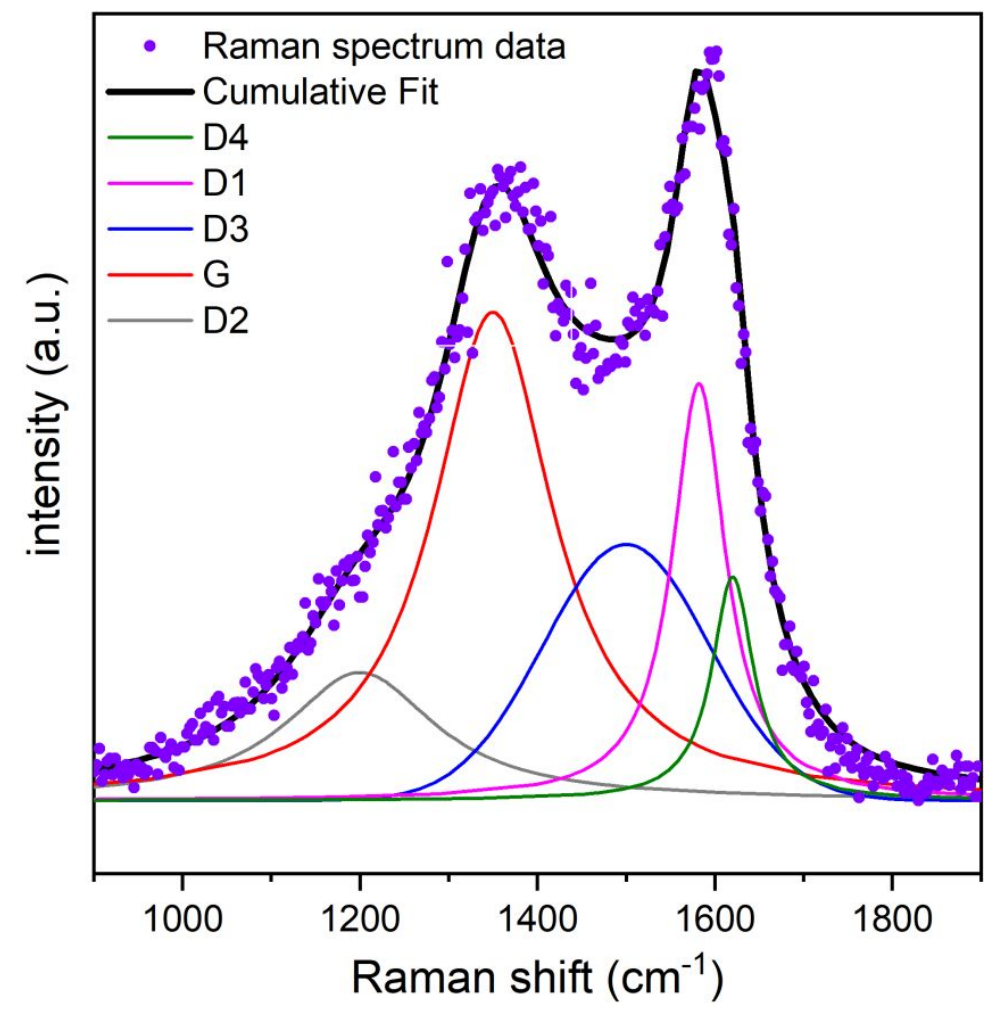

Figure S3. Example of peak decovolution of first order Raman spectrum into five peaks using the method described by Sadezky et al. ${ }^{1}$ Lorentizian line shape fitting used for G, D1, D2, and D4, and Gaussian line shape fitting used for D3.

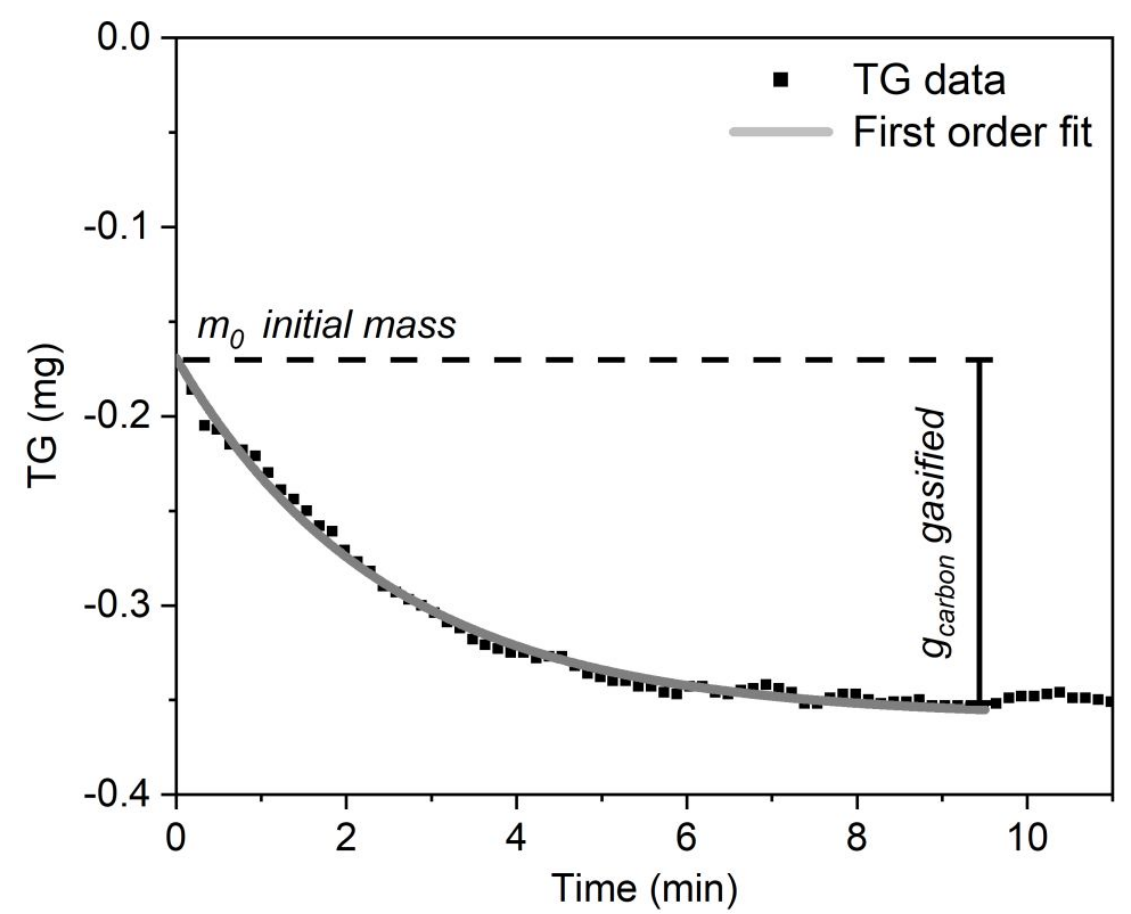

Figure S4. Example of first order fit for TG mass loss profile during gasification with $33 \% \mathrm{H}_{2} \mathrm{O}$ of the in situ coked $\mathrm{MnCr}_{2} \mathrm{O}_{4}$ catalyst at $850{ }^{\circ} \mathrm{C}$. 


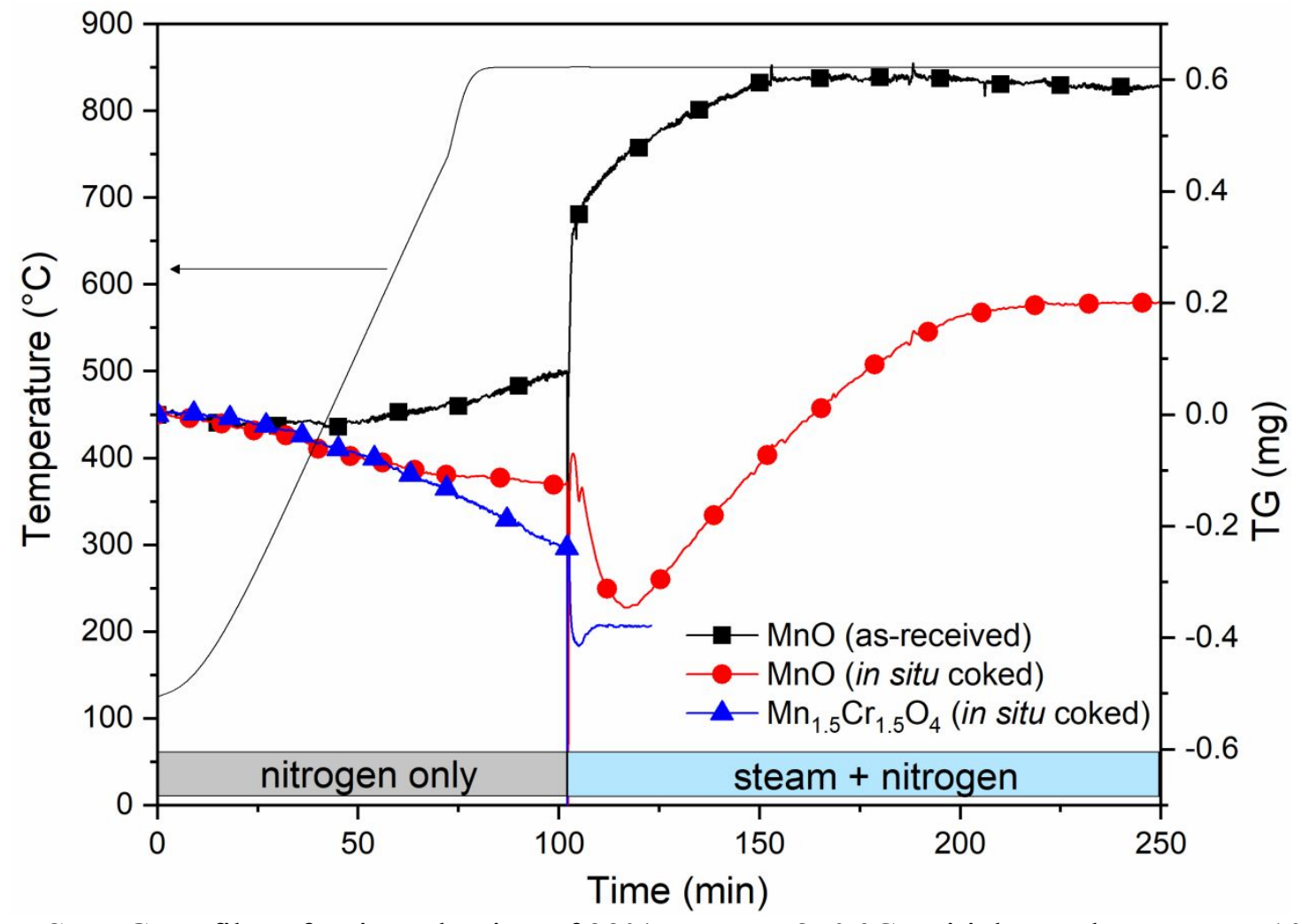

Figure S5. TG profiles after introduction of $33 \%$ steam at $850{ }^{\circ} \mathrm{C}$. Initial sample masses: $\sim 10 \mathrm{mg}$.

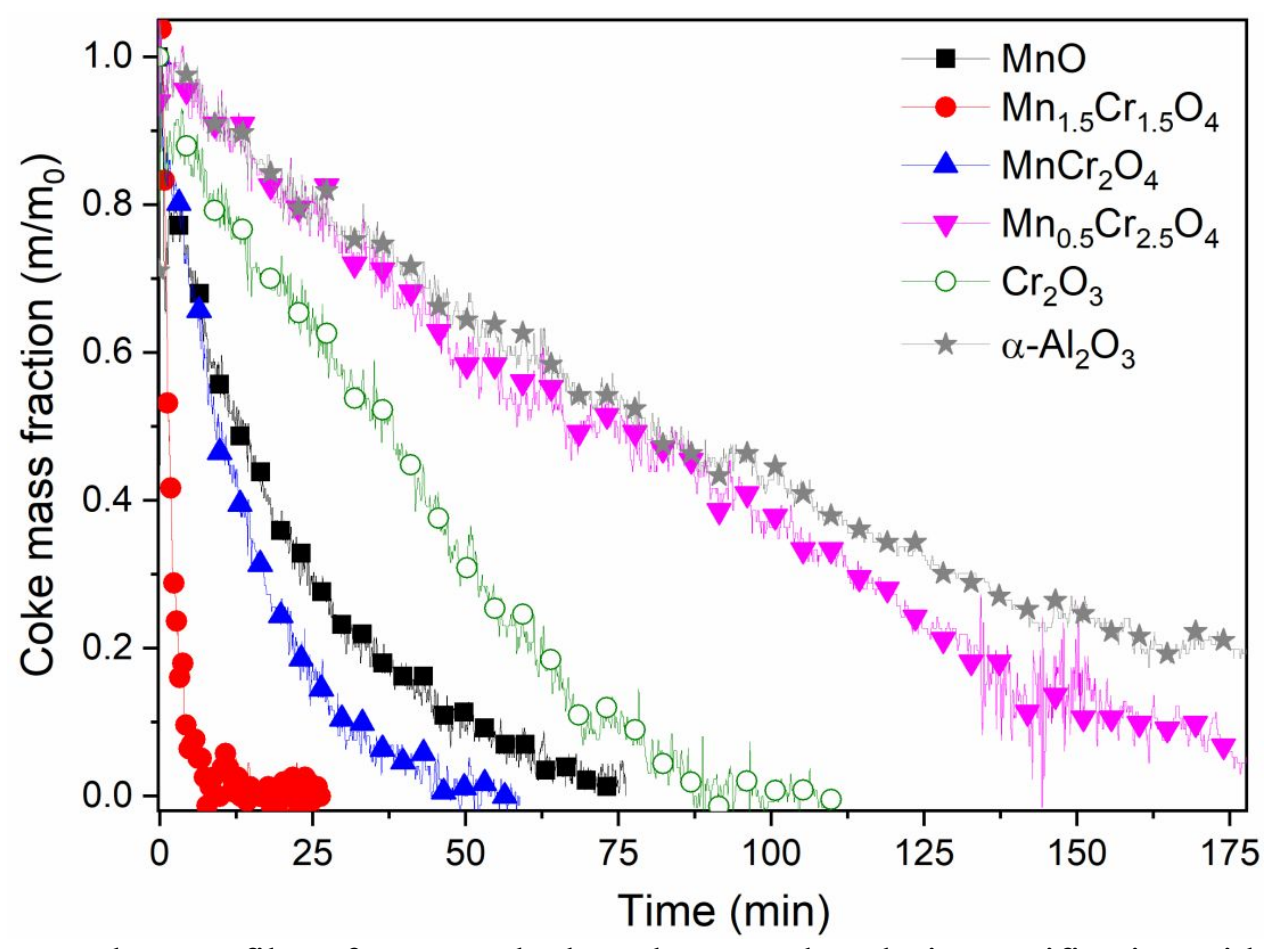

Figure S6. Mass loss profiles of in situ coked catalyst powders during gasification with $18 \% \mathrm{H}_{2} \mathrm{O}$ $2.3 \% \mathrm{H}_{2}$ mixture at $850{ }^{\circ} \mathrm{C}$. 

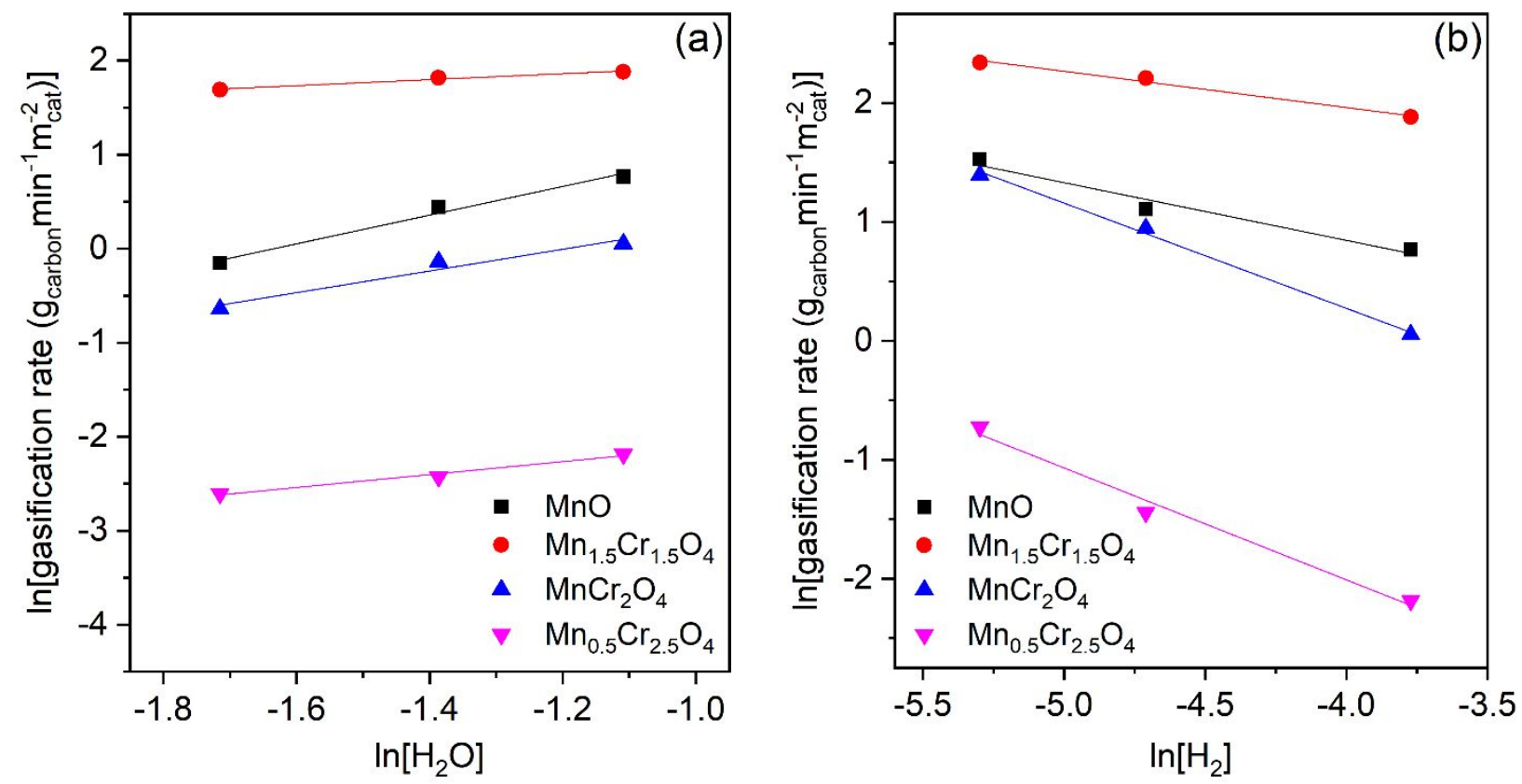

Figure S7. Effect of different (a) $\mathrm{H}_{2} \mathrm{O}$ and (b) $\mathrm{H}_{2}$ concentrations on the coke gasification activity of Mn-Cr-O catalysts, with apparent reaction orders summarized in Table S2. (a) Reaction conditions: $850{ }^{\circ} \mathrm{C}, 18-33 \% \mathrm{H}_{2} \mathrm{O}, 2.3 \% \mathrm{H}_{2}$, balance $\mathrm{N}_{2}$. (b) Reaction conditions: $850{ }^{\circ} \mathrm{C}, 33 \% \mathrm{H}-$ ${ }_{2} \mathrm{O}, 0.5-2.3 \% \mathrm{H}_{2}$, balance $\mathrm{N}_{2}$.

Table S2. Apparent reaction orders for Mn-Cr-O catalysts for coke gasification and comparable reaction data for steam reforming of ethylene from reference. ${ }^{2}$

\begin{tabular}{|c|c|c|c|c|c|}
\hline \multirow[b]{2}{*}{ Oxide Catalyst } & \multicolumn{2}{|c|}{ Coke gasification } & \multicolumn{3}{|c|}{ Ethylene steam reforming ${ }^{2}$} \\
\hline & $\begin{array}{c}\text { Order in } \\
\mathrm{H}_{2} \mathrm{O}\end{array}$ & $\begin{array}{c}\text { Order in } \\
\mathrm{H}_{2}\end{array}$ & $\begin{array}{c}\text { Order in } \\
\mathrm{H}_{2} \mathrm{O}\end{array}$ & $\begin{array}{c}\text { Order in } \\
\mathrm{H}_{2}\end{array}$ & $\begin{array}{c}\mathrm{C}_{1} \text { production rate } \\
\left(\mu \mathrm{mol} \mathrm{m} \mathrm{m}^{-2} \mathrm{~s}^{-1}\right)\end{array}$ \\
\hline $\mathrm{MnO}$ & 1.5 & -0.49 & -- & -- & 0.060 \\
\hline $\mathrm{Mn}_{1.5} \mathrm{Cr}_{1.5} \mathrm{O}_{4}$ & 0.32 & -0.30 & -0.40 & -0.05 & 0.21 \\
\hline $\mathrm{MnCr}_{2} \mathrm{O}_{4}$ & 1.1 & -0.90 & -0.21 & 0.03 & 0.13 \\
\hline $\mathrm{Mn}_{0.5} \mathrm{Cr}_{2.5} \mathrm{O}_{4}$ & 0.70 & -0.94 & -0.38 & -0.05 & 0.19 \\
\hline
\end{tabular}

${ }^{a}$ Reaction conditions: $600{ }^{\circ} \mathrm{C}, 25 \% \mathrm{C}_{2} \mathrm{H}_{4}, 50 \% \mathrm{H}_{2} \mathrm{O}$, bal. inert.

\section{References}

(1) Sadezky, A.; Muckenhuber, H.; Grothe, H.; Niessner, R.; Pöschl, U. Raman Microspectroscopy of Soot and Related Carbonaceous Materials: Spectral Analysis and Structural Information. Carbon N. Y. 2005, 43 (8), 1731-1742. https://doi.org/10.1016/j.carbon.2005.02.018.

(2) Yang, L.; Bukhovko, M. P.; Brezicki, G.; Malek, A.; Li, L.; Jones, C. W.; Agrawal, P. K.; Davis, R. J. Steam Reforming of Ethylene over Manganese-Chromium Spinel Oxides. $J$. Catal. 2019, 380, 224-235. https://doi.org/https://doi.org/10.1016/j.jcat.2019.10.006. 